Article

\title{
An Explicit Expression of Average Run Length of Exponentially Weighted Moving Average Control Chart with ARIMA $(p, d, q)(P, D, Q)_{L}$ Models
}

\author{
Yupaporn Areepong * and Saowanit Sukparungsee \\ Department of Applied Statistics, Faculty of Applied Science, King Mongkut's University of Technology \\ North Bangkok, 1518 Pracharat 1 road, Wongsawang, Bangsue, Bangkok 10800, Thailand ; \\ saowanit.s@sci.kmutnb.ac.th \\ * Correspondence: yupaporn.a@sci.kmutnb.ac.th; Tel.: +66-2-555-2000
}

\begin{abstract}
In this paper we propose the explicit formulas of Average Run Length $(A R L)$ of Exponentially Weighted Moving Average (EWMA) control chart for Autoregressive Integrated Moving Average: ARIMA (p,d,q) (P, D, Q) t process with exponential white noise. To check the accuracy, the ARL results were compared with numerical integral equations based on the Gauss-Legendre rule. There was an excellent agreement between the explicit formulas and the numerical solutions. Additionally, we compared the computational time between our explicit formulas for the ARL with the one obtained via Gauss-Legendre numerical scheme. The computational time for the explicit formulas was approximately one second that is much less than the numerical approximations. The explicit analytical formulas for evaluating ARL0 and ARL1 can produce a set of optimal parameters which depend on the smoothing parameter $(\lambda)$ and the width of control limit $(H)$, for designing an EWMA chart with a minimum ARL1.
\end{abstract}

Keywords: exponentially weighted moving average control chart (EWMA); autoregressive integrated moving average (ARIMA); average run length (ARL)

\section{Introduction}

A control chart is an effective tool in statistical process control for detecting changes in a mean or variance processes and can be used for measuring, controlling and improving quality in area such as industrial statistics and manufacturing, financial service, environmental statistics, healthcare, medical research and others; see Lucas and Saccucci [1] and Srivastava and Wu [2]. Many control charts have been developed including the Shewhart, Exponentially Weighted Moving Average (EWMA) and cumulative SUM (CUSUM). In this paper, we discuss the Exponentially Weighted Moving Average (EWMA) chart which is used for detecting small changes of parameters (Roberts [3]; Crowder [4]; Lucas and Saccucci [1]). EWMA control chart was proposed by Roberts [3] in quality control in order to detect a small shift in the mean of a production process as soon as it occurs. Various methods for evaluating the performance of the EWMA procedure have been studied in the literature (see Yashchin [5], Srivastava and Wu [2], Borror et al.[6]). A basic assumption in standard applications of control charts is that observations from the process at different times are independent and identically distributed (i.i.d) random variables. However, in many situations, a process does not yield sufficient observations for traditional SPC tools to be used effectively. However, production process observations often show some autocorrelation. For instance in chemical and continuous industries, wind speeds, the daily flow of a river and the amount of dissolved oxygen in the water are process data which are auto correlated. Several researchers in different fields of study have considered the problem of data correlation and how it relates to SPC. Positive autocorrelation in observations can appear in negative bias in traditional estimators of the standard deviation. This bias produces control limits for standard control charts that are much 
tighter than desired. It has been observed that the main effect of autocorrelation in process data for a traditional chart is that the Average Run Length of the in-control processes may be shorter than intended. Processes with serially correlated data need to be monitored by appropriate control charts.

Two measures that are commonly used to compare the performance of control charts are the Average Run Length for in control process (ARLo) and the Average Run Length for out of control process (ARL1). The ARLo is the average number of observations that will occur before an in-control process falsely gives an out-of-control signal. To reduce the number of false out-of-control signals a sufficiently large ARLo is required. The ARL 1 is a measure of the average number of observations that will occur before an out-of-control process correctly gives an out-of-control signal. To reduce the time that the process is out-of-control, a small ARL1 is required. Therefore the ARL and ARL1 are two conflicting criteria that must be balanced to give an optimal control chart.

Three standard methods that are often used to evaluate Average Run Length for in control process (ARLo) and the Average Run Length for out of control process (ARL1) are the Markov Chain Approach (MCA), the Integral Equation (IE) and the Monte Carlo simulation (MC) methods.

Roberts [3] evaluated the ARL for EWMA control charts using the MC technique. Crowder [4] computed the ARL of an EWMA chart from numerical solutions to an integral for Gaussian observation. Lucas and Saccucci [1] employed a finite state Markov chain approximation to develop tables to assist users of EWMA control charts in these choices.

The methods used for the evaluation of the characteristics of EWMA control charts for serial correlated data were studied. Mastrangelo and Montgomery [7] have been evaluated the performance of EWMA control charts for serially-correlated observation using Monte Carlo simulation technique. Vanbrackle and Reynold [8] studied EWMA and CUSUM control charts by using an Integral Equation and Markov Chain Approach to evaluate the ARL in case of AR(1) process with additional random error. Harris and Ross [9] discussed the effect of autocorrelation on the performance of EWMA and CUSUM charts. They found that the Average Run Lengths and Median Run Lengths of these charts were sensitive to autocorrelation. Later, Reynolds and Lu [10] studied the EWMA control charts using simulation based on the observations from the AR(1) process plus a random error of detecting change in the process mean or variance. Lu and Reynolds [11] presented the ARL of the EWMA control chart based on residual from the forecast value for monitoring the mean of the process for an AR(1) process plus a random error using an integral equation method. Apley and Lee [12] presented a technique for designing residual based EWMA charts under conditions of model uncertainty. Shiau and Chen [13] investigated the robustness of modified individual Shewhart and modified exponentially weighted moving average (EWMA) charts for normality assumption of the white noise term for AR(1) process with positive autocorrelation. Rosolowski and Schmid [14] measured ARL of EWMA charts by monitoring the mean of the stationary processes with heavy tailed distribution using simulation. Mititelu et al. [15] presented explicit formulas for the ARL of EWMA and CUSUM charts when the observations have a hyper exponential distribution, using the Fredholm integral equations approach. Recently, Suriyakat et al. [16] derived an exact solutions of ARL for EWMA control charts for AR(1) process observations with exponential white noise. Busaba et al. [17] have studied an explicit formula of ARL for cumulative sum charts using negative exponential data. Petcharat et al. [18] presented closed form expression of the ARL for CUSUM chart for MA(1) processes with exponential white noise using integral equations. Phanyaem et al. [19] presented Explicit formulas of average run length for $\operatorname{ARMA}(1,1)$ using the Fredholm integral equations approach.

In this research the objective is to derive explicit formulas for detecting changes in the mean of the process of EWMA control charts for ARIMA (p,d,q) (P, D, Q) L Process with exponential white noise. Additionally, the explicit formulas of ARL0 and ARL1 can be able to generate a set of optimal parameters which depend on the smoothing parameter $(\lambda)$ and the width of control limit control $\operatorname{limit}(H)$ for designing EWMA charts with a minimum of ARL1.

\section{EWMA Chart for ARIMA $(\mathrm{p}, \mathrm{d}, \mathrm{q})(\mathrm{P}, \mathrm{D}, \mathrm{Q})_{\mathrm{L}}$ Process and Characteristic}


Let $\xi_{1}, \xi_{2}, \ldots$, be sequentially observed independent random variables with a distribution $F(x, \beta)$ where $\beta$ is a parameter. We study the change-point detection problem, i.e. of detecting if and when the value of the parameter $\beta$ changes. The change-point model for the exponential distribution may be stated as follows. We assume that:

$$
\xi_{t} \sim\left\{\begin{array}{c}
\operatorname{Exp}\left(\beta_{0}\right), \quad t=1,2, \ldots, \theta-1 \\
\operatorname{Exp}\left(\beta_{1}\right), t=\theta, \theta+1, \theta+2, \ldots
\end{array}\right.
$$

where $\beta_{0}$ and $\beta_{1}$ are known parameters. Usually, the parameter value $\beta_{0}$ is assumed to define the in-control state and the parameter value $\beta_{1}$ to denote an out-of-control state. We assume that the value $\beta_{0}$ is maintained up to some unknown time $\theta-1$ and that at time $\theta$ the parameter value changes to the new value $\beta>\beta_{0}$. The time $\theta$ is called "the change-point time".

The typical condition of choice of the stopping times $\tau$ is as follows:

$$
E_{\infty}(\tau)=T,
$$

where $T$ is given (usually large), and $E_{\infty}($.$) denotes that the expectation under distribution$ $F\left(x, \beta_{0}\right)$, 'in-control' is that the change-point occurs at point $\theta$ (where $\theta \leq \infty$ ). In the literature on quality control, the quantity $E_{\infty}(\tau)$ is called the Average Run Length for 'in-control' processes (ARL0). Then, by definition, $A R L_{0}=E_{\infty}(\tau)$ and the typical practical constraint is:

$$
A R L_{0}=E_{\infty}(\tau)=T .
$$

Another common constraint consists of minimizing the quantity:

$$
A R L_{1}=E_{\theta}(\tau-\theta+1 \mid \tau \geq \theta)
$$

where $E_{\theta}($.$) is the expectation under distribution, F\left(x, \beta_{1}\right)$ 'out-of-control' and $\beta$ is the value of the parameter after the change-point. There is restriction on the special case, usually $\theta=1$. The quantity $E_{1}(\tau)$ is called the Average Run Length for 'out-of-control' processes (ARL1) and it could be expected that a sequential chart would have a near optimal performance if ARL1 is close to minimal value.

The definition of EWMA statistics based on ARIMA $(p, d, q)(P, D, Q) L$ process is the following recursion:

$$
Z_{t}=(1-\lambda) Z_{t-1}+\lambda X_{t} ; t=1,2, \ldots .
$$

where $Z_{t}$ is the EWMA statistic, $X_{t}$ is a sequence of ARIMA $(\mathrm{p}, \mathrm{d}, \mathrm{q})(\mathrm{P}, \mathrm{D}, \mathrm{Q}) \mathrm{L}$ process, $\lambda$ is a smoothing parameter, and the initial value is a constant $\left(Z_{0}=\mathrm{u}\right)$.

The general autoregressive processes denoted by ARIMA $(\mathrm{p}, \mathrm{d}, \mathrm{q})(\mathrm{P}, \mathrm{D}, \mathrm{Q}) \mathrm{L}$ process can be written as:

$$
\begin{gathered}
(1-B)^{\mathrm{d}}\left(1-\mathrm{B}^{\mathrm{L}}\right)^{\mathrm{D}}\left(1-\phi_{1} \mathrm{~B}-\phi_{2} \mathrm{~B}^{2}-\ldots-\phi_{\mathrm{P}} \mathrm{B}^{\mathrm{P}}\right)\left(1-\phi_{\mathrm{L}} \mathrm{B}^{\mathrm{L}}-\phi_{2 \mathrm{~L}} \mathrm{~B}^{2 \mathrm{~L}}-\ldots-\phi_{\mathrm{PL}} \mathrm{B}^{\mathrm{PL}}\right) \mathrm{X}_{\mathrm{t}} \\
\theta_{0}+\left(1-\theta_{1} \mathrm{~B}-\theta_{2} \mathrm{~B}^{2}-\ldots-\theta_{\mathrm{q}} \mathrm{B}^{\mathrm{q}}\right)\left(1-\theta_{\mathrm{L}} \mathrm{B}^{\mathrm{L}}-\theta_{2 \mathrm{~L}} \mathrm{~B}^{2 \mathrm{~L}}-\ldots-\theta_{\mathrm{qL}} \mathrm{B}^{\mathrm{qL}}\right) \xi_{\mathrm{t}}
\end{gathered}
$$

where $\xi_{t}$ are independent and identically distributed observed sequences of Exponential distribution. The initial value $\xi_{0}=1$, an autoregressive coefficient $-1 \leq \phi_{i} \leq 1$ and a moving 
average coefficient $-1 \leq \theta_{i} \leq 1$, a seasonal autoregressive coefficient $-1 \leq \phi_{i L} \leq 1$ and a seasonal moving average coefficient $-1 \leq \theta_{i L} \leq 1$. It is assumed the initial value of ARIMA $(\mathrm{p}, \mathrm{d}, \mathrm{q})(\mathrm{P}, \mathrm{D}, \mathrm{Q}) \mathrm{L}$ process equal to 1 .

The first passage time of an EWMA chart is denoted by:

$$
\tau_{H}=\inf \left\{t \geq 0: Z_{t} \geq H\right\},
$$

where $H$ is constant parameter known as the upper control limit.

\section{ARL Explicit Formulas for ARIMA (p,d,q)(P, D, Q) L Process of EWMA Chart}

In this section, we derive explicit solution of Fredholm Integral Equation of the second kind which is called ARL explicit formulas of EWMA chart for ARIMA (p,d,q)(P, D, Q) L process.

Let $L(u)$ denote the ARL of a one-sided EWMA control chart when the initial value is $u$, $Z_{0}=u$. Since $\xi_{t} \geq 0$ we can assume that the lower and upper limits are $H_{L}=0$ and $H_{U}=H$ respectively. For the EWMA statistics $Z_{1}$ in an in-control state:

$$
0<(1-\lambda) Z_{t-1}+\lambda X_{t}<H .
$$

Then the function $L(u)$ is defined as follows:

$$
L(u)=\mathbb{E}_{\infty}(\tau) \geq T, Z_{0}=u .
$$

To consider the function $L(u)$ :

$$
L(u)=1+\int L\left(Z_{1}\right) f\left(\xi_{1}\right) d \xi_{1} .
$$

Equation 7 is a Fredholm integral equation of the second kind.

Consequently the function $L(u)$ is obtained as:

$$
L(u)=1+\int L\left((1-\lambda) u+\lambda X_{t}\right) f(y) d y .
$$

Changing the integration variable, the function $L(u)$ is given by:

$$
L(u)=1+\frac{1}{\lambda} \int_{0}^{H} L(y) f\left(\frac{y-(1-\lambda) u}{\lambda}-X_{t}\right) d y
$$

Therefore, we obtain

$$
L(u)=\frac{1}{\lambda \beta} \int_{0}^{H} L(y) e^{-\frac{y}{\lambda \beta}} e^{\left(\frac{(1-\lambda) u}{\lambda \beta}+\frac{X_{t}}{\beta}\right)} d y .
$$

Let the function $G(u)$ be given by:

$$
G(u)=e^{\left(\frac{(1-\lambda) u}{\lambda \beta}+\frac{X_{t}}{\beta}\right)} .
$$

Consequently,

$$
L(u)=1+\frac{G(u)}{\lambda \beta} \int_{0}^{H} L(y) e^{-\frac{y}{\lambda \beta}} d y \quad ; 0 \leq u \leq H .
$$

Let $k=\int_{0}^{H} L(y) e^{-\frac{y}{\lambda \beta}} d y, \quad$ so we have; $L(u)=1+\frac{G(u)}{\lambda \beta} k$.

Therefore, we obtain 


$$
L(u)=1+\frac{1}{\lambda \beta} e^{\left(\frac{(1-\lambda) u}{\lambda \beta}+\frac{X_{t}}{\beta}\right)} k
$$

Solving a constant $k$;

$$
k=\frac{(-\lambda \beta)\left(e^{-\frac{H}{\lambda \beta}}-1\right)}{1+\frac{1}{\lambda} e^{\left(\frac{X_{t}}{\beta}\right)}\left(e^{-\frac{H}{\beta}}-1\right)} .
$$

Substituting $k$ into Eq. (9) thus the function $L(u)$ can be written as

$$
L(u)=1-\frac{e^{\left(\frac{(1-\lambda) u}{\lambda \beta}+\frac{X_{t}}{\beta}\right)}\left(e^{-\frac{H}{\lambda \beta}}-1\right)}{1+\frac{1}{\lambda} e^{\left(\frac{X_{t}}{\beta}\right)}\left(e^{-\frac{H}{\beta}}-1\right)} .
$$

As mentioned above, the value of the parameter $\beta$ is equal to $\beta_{0}$ when the process is in control process. Therefore, substituting $\beta=\beta_{0}$ into Eq. (10) gives the formula for the ARLo as:

$$
A R L_{0}=1-\frac{e^{\left(\frac{(1-\lambda) u}{\lambda \beta_{0}}+\frac{X_{t}}{\beta_{0}}\right)}\left(e^{-\frac{H}{\lambda \beta_{0}}}-1\right)}{1+\frac{1}{\lambda} e^{\left(\frac{X_{t}}{\beta_{0}}\right)}\left(e^{-\frac{H}{\beta_{0}}}-1\right)} .
$$

The formula for ARL1 can be obtained in a similar manner. When the process is out of control process the value of the parameter $\beta$ in Eq. (11) will be $\beta=\beta_{1}$. The formula for ARL1 can therefore be written as:

$$
A R L_{1}=1-\frac{e^{\left(\frac{(1-\lambda) u}{\lambda \beta_{1}}+\frac{X_{t}}{\beta_{1}}\right)}\left(e^{-\frac{H}{\lambda \beta_{1}}}-1\right)}{1+\frac{1}{\lambda} e^{\left(\frac{X_{t}}{\beta_{1}}\right)}\left(e^{-\frac{H}{\beta_{1}}}-1\right)}
$$

where $X_{t}$ is a sequence of $\operatorname{ARIMA}(\mathrm{p}, \mathrm{d}, \mathrm{q})(\mathrm{P}, \mathrm{D}, \mathrm{Q})$ L process, $\lambda \in(0,1)$ is the smoothing parameter, $u$ is the initial values and $H$ is the upper control limit.

According to Equation 11 and 12, for example, the explicit formulas of ARL, for example

$\operatorname{ARIMA}(1,1,1)(0,1,1)$ L process can be written $X_{t}$ as

$$
X_{t}=\mu+\xi_{t}-\theta_{1} \xi_{t-1}-\theta_{L} \xi_{t-L}+\theta_{1} \theta_{L} \xi_{t-(1+L)}+X_{t-L}+X_{t-1}-X_{t-(1+L)}+\phi_{1} X_{t-1}-\phi_{1} X_{t-(1+L)}-\phi_{1} X_{t-2}+\phi_{1} X_{t-(2+L)}
$$

$\operatorname{ARIMA}(0,1,2)(1,1,2)$ L process can be written $X_{t}$ as

$$
\begin{aligned}
X_{t}= & \mu+\xi_{t}-\theta_{1} \xi_{t-1}-\theta_{2} \xi_{t-2}-\theta_{L} \xi_{t-L}+\theta_{1} \theta_{2} \xi_{t+L}+\theta_{2} \theta_{L} \xi_{t-(2+L)}-\theta_{2 L} \xi_{t-2 L}+\theta_{1} \theta_{2 L} \xi_{t-(1+2 \mathrm{~L})} \\
& +X_{t-L}+X_{t-1}-X_{t-(1+L)}+\phi_{L} X_{t-L}-\phi_{L} X_{t-2 L}-\phi_{L} X_{t-(1+L)}+\phi_{L} X_{t-(1+2 L)}
\end{aligned}
$$

$\operatorname{ARIMA}(0,1,1)(1,0,2)$ L process can be written $X_{t}$ as

$$
X_{t}=\mu+\xi_{t}-\theta_{1} \xi_{t-1}-\theta_{L} \xi_{t-L}+\theta_{1} \theta_{L} \xi_{t-(1+L)}-\theta_{2 L} \xi_{t-2 L}+\theta_{1} \theta_{2 L} \xi_{t-(1+2 \mathrm{~L})}+X_{t-1}+\phi_{L} X_{t-L}-\phi_{L} X_{t-(1+L)}
$$

Using the explicit formulas in Equation 11 and 12, we can provide the tables for the optimal smoothing parameter $(\lambda)$ and width of control limit $(H)$ for designing EWMA chart with minimum of ARL1. We firstly describe a procedure for obtaining optimal designs for EWMA chart. The criterions for choosing optimal values are smoothing parameter $(\lambda)$ and width of control limit $(H)$ for designing EWMA chart with minimum of ARL1 for a given in-control parameter value $\beta_{0}=1, A R L_{0}=T$ and a given out-of-control parameter value $\left(\beta=\beta_{1}\right)$. We compute optimal 
$(\lambda, H)$ values for $\mathrm{T}=370$ and magnitudes of change. Table of the optimal parameters values are shown in Table 4.

The numerical procedure for obtaining optimal parameters for EWMA designs

1. To select an acceptable in-control value of ARL and decide on the change parameter value $\left(\beta_{1}\right)$ for an out-of-control process.

2. For given $\beta_{0}$ and $\mathrm{T}$, find optimal values of $\lambda$ and $H$ to minimize the $\mathrm{ARL}_{1}\left(\mathrm{ARL}_{1}{ }^{*}\right.$ ) values given by equation 12 subject to the constraint that $\mathrm{ARL}_{0}=\mathrm{T}$ in Equation 11, i.e. $\lambda$ and $H$ are solutions of the optimality problem

\section{Numerical Results}

In this section, we compare the results of $\mathrm{ARL}_{0}$ and $\mathrm{ARL}_{1}$ for $\operatorname{ARIMA}(\mathrm{p}, \mathrm{d}, \mathrm{q})(\mathrm{P}, \mathrm{D}, \mathrm{Q}) \mathrm{L}$ process which obtained from the explicit formulas with numerical solution of integral equation method for the number of division point $\mathrm{m}=500$. A numerical scheme to evaluate solution of the integral equations (IE) is given by

$$
\tilde{L}(u)=1+\tilde{L}\left(a_{1}\right) F\left(a-u-X_{t}\right)+\sum_{j=1}^{m} w_{j} \tilde{L}\left(a_{j}\right) f\left(a_{j}+a-u-X_{t}\right) .
$$

where $a_{j}=\frac{H}{m}\left(j-\frac{1}{2}\right)$ and $w_{j}=\frac{H}{m} ; j=1,2, \ldots, m$.

The results of ARL are presented in Table 1 - Table 3. The parameter values for EWMA chart were chosen by given desired $\mathrm{ARL}_{0}=370$ and 500 , in-control parameter $\beta_{0}=1$ and magnitudes of change. We consider the performance of the explicit formulas in term of the computational times and the absolute percentage difference can be computed as follows:

$$
\operatorname{Diff}(\%)=\frac{\left|A R L_{\text {Explicit Formulas }}-A R L_{\text {Numerical IE }}\right|}{A R L_{\text {Explicit Formulas }}} \times 100 .
$$

We compare the numerical results for ARLo and ARL for Exponential (1) obtained from explicit formulas with results obtained from the Integral Equation method for parameter values $\lambda=0.05$. The table shows that the outputs obtained by explicit formulas are very close to IE results. The choice of method for calculating ARL values should therefore be made based on other factors (e.g. CPU times, available software or programming). However, the table also shows that the computational time for evaluating the suggested formula is much less than the CPU times required for IE method. The numerical results in terms of optimal EWMA smoothing parameter $(\lambda)$ and width of control limit $(H)$ and minimal of ARL1 are shown in Table 4. For example, if we want to detect a parameter change from $\beta_{0}=1$ to $\beta_{1}=1.05$ and the ARL value is $\mathrm{T}=370$, then the optimality procedure given above will give optimal parameter values $\lambda=0.01$ and $H=0.00527571$. On substituting the values for $\beta, \lambda$ and $H$ into Equation 12 we obtain an optimal ARL1 value of $\mathrm{ARL}_{1}^{*}=10.31$. 
Table 1. Comparison of ARL values for ARIMA $(1,1,1)(0,1,1) 12$ using explicit formula against NIE when $\lambda=0.05 \phi_{1}=0.2, \theta_{1}=0.2, \theta_{12}=0.2$

\begin{tabular}{|c|c|c|c|c|c|c|}
\hline \multirow{3}{*}{$\beta$} & \multicolumn{3}{|c|}{$\mathrm{ARL}_{0}=370$} & \multicolumn{3}{|c|}{$\mathrm{ARL}_{0}=500$} \\
\hline & \multicolumn{2}{|c|}{$H=0.0266609$} & \multirow[t]{2}{*}{ Diff (\%) } & \multicolumn{2}{|c|}{$H=0.0266759$} & \multirow[t]{2}{*}{ Diff $(\%)$} \\
\hline & Explicit & Numerical IE & & Explicit & Numerical IE & \\
\hline \multirow{2}{*}{1.00} & 370.101 & & & 500.589 & 500.589 & \\
\hline & $(0.14)$ & $370.1009(17.682)$ & 0.00003 & $(0.14)$ & $(18.361)$ & 0.00000 \\
\hline \multirow{2}{*}{1.01} & 43.6705 & 43.6705 & & 45.0132 & 45.0132 & \\
\hline & $(0.14)$ & $(17.821)$ & 0.00000 & $(0.14)$ & $(18.429)$ & 0.00000 \\
\hline \multirow{2}{*}{1.02} & 23.6665 & 23.6665 & & 24.0439 & 24.0439 & \\
\hline & $(0.14)$ & (18.116) & 0.00000 & $(0.14)$ & (18.617) & 0.00000 \\
\hline \multirow{2}{*}{1.03} & 16.4421 & 16.4421 & & 16.6181 & 16.6181 & \\
\hline & $(0.14)$ & $(18.372)$ & 0.00000 & $(0.14)$ & (19.015) & 0.00000 \\
\hline \multirow{2}{*}{1.04} & 12.7154 & 12.7154 & & 12.8175 & 12.8175 & \\
\hline & $(0.14)$ & $(17.757)$ & 0.00000 & $(0.14)$ & (18.447) & 0.00000 \\
\hline \multirow{2}{*}{1.05} & 10.4413 & 10.4413 & & 10.5082 & 10.5082 & \\
\hline & $(0.14)$ & $(18.522)$ & 0.00000 & $(0.14)$ & $(18.603)$ & 0.00000 \\
\hline \multirow{2}{*}{1.06} & 8.90899 & 8.90898 & & 8.95634 & 8.95633 & \\
\hline & $(0.14)$ & (17.734) & 0.00011 & $(0.14)$ & (18.738) & 0.00011 \\
\hline \multirow{2}{*}{1.07} & 7.8063 & 7.8063 & & 7.84171 & 7.8417 & \\
\hline & $(0.14)$ & (18.142) & 0.00000 & $(0.14)$ & $(18.862)$ & 0.00013 \\
\hline \multirow{2}{*}{1.08} & 6.97476 & 6.97476 & & 7.0023 & 7.0023 & \\
\hline & $(0.14)$ & $(18.469)$ & 0.00000 & $(0.14)$ & (19.026) & 0.00000 \\
\hline \multirow{2}{*}{1.09} & 6.32527 & 6.32527 & & 6.34736 & 6.34736 & \\
\hline & $(0.14)$ & (18.718) & 0.00000 & $(0.14)$ & $(18.761)$ & 0.00000 \\
\hline \multirow{2}{*}{1.10} & 5.80392 & 5.80392 & & 5.82207 & 5.82207 & \\
\hline & $(0.14)$ & $(17.972)$ & 0.00000 & $(0.14)$ & (19.152) & 0.00000 \\
\hline \multirow{2}{*}{1.30} & 2.63948 & 2.63948 & & 2.64204 & 2.64203 & \\
\hline & $(0.14)$ & (18.438) & 0.00000 & $(0.14)$ & (19.384) & 0.00038 \\
\hline \multirow{2}{*}{1.50} & 1.99607 & 1.99607 & & 1.99719 & 1.99719 & \\
\hline & $(0.14)$ & $(19.216)$ & 0.00000 & $(0.14)$ & $(19.493)$ & 0.00000 \\
\hline
\end{tabular}

( ) Computational Time (Sec.) 
Table 2. Comparison of ARL values for ARIMA $(0,1,2)(1,1,2) 6$ using explicit formula against NIE when $\lambda=0.05 \phi_{6}=0.1, \theta_{1}=0.1, \theta_{2}=0.1, \theta_{6}=0.1, \theta_{12}=0.1$

\begin{tabular}{|c|c|c|c|c|c|c|}
\hline \multirow{3}{*}{$\beta$} & \multicolumn{3}{|c|}{$\mathrm{ARL}_{0}=370$} & \multicolumn{3}{|c|}{$\mathrm{ARL}_{0}=500$} \\
\hline & \multicolumn{2}{|c|}{$H=0.0266609$} & \multirow[t]{2}{*}{ Diff $(\%)$} & \multicolumn{2}{|r|}{$H=0.0266759$} & \multirow[t]{2}{*}{$\operatorname{Diff}(\%)$} \\
\hline & Explicit & Numerical IE & & Explicit & Numerical IE & \\
\hline \multirow{2}{*}{1.00} & 370.207 & 370.207 & & 500.26 & 500.259 & \\
\hline & $(0.14)$ & $(18.627)$ & 0.00000 & $(0.14)$ & $(19.427)$ & 0.00020 \\
\hline \multirow{2}{*}{1.01} & 42.8521 & 42.8521 & & 44.1401 & 44.1401 & \\
\hline & $(0.14)$ & $(18.751)$ & 0.00000 & $(0.14)$ & $(19.513)$ & 0.00000 \\
\hline \multirow{2}{*}{1.02} & 23.1993 & 23.1993 & & 23.5606 & 23.5606 & \\
\hline & $(0.14)$ & $(18.941)$ & 0.00000 & $(0.14)$ & $(19.732)$ & 0.00000 \\
\hline \multirow{2}{*}{1.03} & 16.1131 & 16.1131 & & 16.2815 & 16.2815 & \\
\hline & $(0.14)$ & (19.203) & 0.00000 & $(0.14)$ & $(19.846)$ & 0.00000 \\
\hline \multirow{2}{*}{1.04} & 12.4602 & 12.4602 & & 12.5578 & 12.5578 & \\
\hline & $(0.14)$ & (19.378) & 0.00000 & $(0.14)$ & $(20.018)$ & 0.00000 \\
\hline \multirow{2}{*}{1.05} & 10.2319 & 10.2319 & & 10.2958 & 10.2958 & \\
\hline & $(0.14)$ & (19.504) & 0.00000 & $(0.14)$ & $(20.273)$ & 0.00000 \\
\hline \multirow{2}{*}{1.06} & 8.73071 & 8.73071 & & 8.77601 & 8.77601 & \\
\hline & $(0.14)$ & (19.665) & 0.00000 & $(0.14)$ & (19.785) & 0.00000 \\
\hline \multirow{2}{*}{1.07} & 7.65069 & 7.65068 & & 7.68455 & 7.68455 & \\
\hline & $(0.14)$ & (19.737) & 0.00013 & $(0.14)$ & $(20.262)$ & 0.00000 \\
\hline \multirow{2}{*}{1.08} & 6.83634 & 6.83634 & & 6.86269 & 6.86269 & \\
\hline & $(0.14)$ & (18.823) & 0.00000 & $(0.14)$ & (19.925) & 0.00000 \\
\hline \multirow{2}{*}{1.09} & 6.20036 & 6.20036 & & 6.22149 & 6.22149 & \\
\hline & $(0.14)$ & (19.138) & 0.00000 & $(0.14)$ & (20.328) & 0.00000 \\
\hline \multirow{2}{*}{1.10} & 5.68991 & 5.6899 & & 5.70728 & 5.70727 & \\
\hline & $(0.14)$ & (19.269) & 0.00018 & $(0.14)$ & $(20.273)$ & 0.00018 \\
\hline \multirow{2}{*}{1.30} & 2.5933 & 2.5933 & & 2.59575 & 2.59574 & \\
\hline & $(0.14)$ & (18.872) & 0.00000 & $(0.14)$ & (19.877) & 0.00038 \\
\hline \multirow{2}{*}{1.50} & 1.96475 & 1.96475 & & 1.96582 & 1.96582 & \\
\hline & $(0.14)$ & $(19.212)$ & 0.00000 & $(0.14)$ & $(20.351)$ & 0.00000 \\
\hline
\end{tabular}


Table 3. Comparison of ARL values for ARIMA $(0,1,1)(1,0,2) 4$ using explicit formula against NIE when $\lambda=0.05 \theta_{1}=0.1, \theta_{4}=0.2, \theta_{8}=0.3$

\begin{tabular}{|c|c|c|c|c|c|c|}
\hline \multirow{3}{*}{$\beta$} & \multicolumn{3}{|c|}{$\mathrm{ARL}_{0}=370$} & \multirow{2}{*}{\multicolumn{2}{|c|}{$\begin{array}{c}\text { ARL } 0=500 \\
H=0.0266759\end{array}$}} & \multirow{3}{*}{$\operatorname{Diff}(\%)$} \\
\hline & \multicolumn{2}{|c|}{$H=0.0266609$} & \multirow[t]{2}{*}{ Diff (\%) } & & & \\
\hline & Explicit & Numerical IE & & Explicit & Numerical IE & \\
\hline \multirow{2}{*}{1.00} & 370.359 & 370.359 & & 500.202 & 500.202 & \\
\hline & $(0.14)$ & (18.783) & 0.00000 & $(0.14)$ & (17.588) & 0.00000 \\
\hline \multirow{2}{*}{1.01} & 46.745 & 46.745 & & 48.281 & 48.281 & \\
\hline & $(0.14)$ & (17.466) & 0.00000 & $(0.14)$ & (16.24) & 0.00000 \\
\hline \multirow{2}{*}{1.02} & 25.428 & 25.428 & & 25.863 & 25.863 & \\
\hline & $(0.14)$ & (14.196) & 0.00000 & $(0.14)$ & $(18.954)$ & 0.00000 \\
\hline \multirow{2}{*}{1.03} & 17.684 & 17.684 & & 17.887 & 17.887 & \\
\hline & $(0.14)$ & (16.879) & 0.00000 & $(0.14)$ & $(18.606)$ & 0.00000 \\
\hline \multirow{2}{*}{1.04} & 13.679 & 13.679 & & 13.797 & 13.798 & \\
\hline & $(0.14)$ & (19.531) & 0.00000 & $(0.14)$ & $(18.289)$ & 0.00000 \\
\hline \multirow{2}{*}{1.05} & 11.233 & 11.233 & & 11.310 & 11.310 & \\
\hline & $(0.14)$ & (18.199) & 0.00000 & $(0.14)$ & $(16.941)$ & 0.00000 \\
\hline \multirow{2}{*}{1.06} & 9.583 & 9.583 & & 9.637 & 9.637 & \\
\hline & $(0.14)$ & $(18.82)$ & 0.00000 & $(0.14)$ & (19.64) & 0.00000 \\
\hline \multirow{2}{*}{1.07} & 8.394 & 8.394 & & 8.435 & 8.435 & \\
\hline & $(0.14)$ & $(17.472)$ & 0.00000 & $(0.14)$ & (19.385) & 0.00000 \\
\hline \multirow{2}{*}{1.08} & 7.497 & 7.497 & & 7.529 & 7.529 & \\
\hline & $(0.14)$ & (18.108) & 0.00000 & $(0.14)$ & (19.069) & 0.00000 \\
\hline \multirow{2}{*}{1.09} & 6.797 & 6.797 & & 6.823 & 6.823 & \\
\hline & $(0.14)$ & (62.792) & 0.00000 & $(0.14)$ & $(17.736)$ & 0.00000 \\
\hline \multirow{2}{*}{1.10} & 6.234 & 6.234 & & 6.255 & 6.255 & \\
\hline & $(0.14)$ & (16.475) & 0.00000 & $(0.14)$ & (18.451) & 0.00000 \\
\hline \multirow{2}{*}{1.30} & 2.814 & 2.814 & & 2.817 & 2.817 & \\
\hline & $(0.14)$ & (18.111) & 0.00000 & $(0.14)$ & (17.087) & 0.00000 \\
\hline \multirow{2}{*}{1.50} & 2.114 & 2.114 & & 2.116 & 2.116 & \\
\hline & $(0.14)$ & (17.857) & 0.00000 & $(0.14)$ & $(16.802)$ & 0.00000 \\
\hline
\end{tabular}

( ) Computational Time (Sec.) 
Table 4. Optimal design parameters and $\mathrm{ARL}_{1}{ }^{*}$ for EWMA chart given $\beta_{0}=1, \mathrm{ARL} \mathrm{E}_{0}=370$.

\begin{tabular}{cccccccc}
\hline & \multicolumn{3}{c}{ ARIMA $(1,1,1),(0,1,1) 12$} & & \multicolumn{3}{c}{ ARIMA $(0,1,2),(1,1,2) 12$} \\
\cline { 2 - 3 } \cline { 7 - 8 }$\beta$ & $\lambda$ & $H$ & ARL1 $^{*}$ & $\beta$ & $\lambda$ & $H$ & ARL1 $^{*}$ \\
\hline 1.01 & 0.01 & 0.00527571 & 43.125 & 1.01 & 0.01 & 0.00498754 & 42.350 \\
1.02 & 0.01 & 0.00527571 & 23.360 & 1.02 & 0.01 & 0.00498754 & 22.918 \\
1.03 & 0.01 & 0.00527571 & 16.230 & 1.03 & 0.01 & 0.00498754 & 15.918 \\
1.04 & 0.01 & 0.00527571 & 12.553 & 1.04 & 0.01 & 0.00498754 & 12.311 \\
1.05 & 0.01 & 0.00527571 & 10.310 & 1.05 & 0.01 & 0.00498754 & 10.111 \\
1.06 & 0.01 & 0.00527571 & 8.799 & 1.06 & 0.01 & 0.00498754 & 8.630 \\
1.07 & 0.01 & 0.00527571 & 7.712 & 1.07 & 0.01 & 0.00498754 & 7.564 \\
1.08 & 0.01 & 0.00527571 & 6.891 & 1.08 & 0.01 & 0.00498754 & 6.760 \\
1.09 & 0.01 & 0.00527571 & 6.251 & 1.09 & 0.01 & 0.00498754 & 6.133 \\
1.10 & 0.01 & 0.00527571 & 5.737 & 1.10 & 0.01 & 0.00498754 & 5.629 \\
1.30 & 0.01 & 0.00527571 & 2.618 & 1.30 & 0.01 & 0.00498754 & 2.573 \\
1.50 & 0.01 & 0.00527571 & 1.983 & 1.50 & 0.01 & 0.00498754 & 1.953 \\
\hline
\end{tabular}

\section{Conclusions}

We have presented the explicit formulas for Average Run Length of EWMA chart for Autoregressive Integrated Moving Average: ARIMA (p,d,q)(P, D, Q) L process for the case of an exponential white noise. We have shown that the proposed explicit formulas are easy to calculate and program. The explicit formulas obviously take the computational time much less than Numerical Integral Equation method.

Acknowledgments: The authors would like to express my gratitude to King Mongkut's University of Technology North Bangkok and the Office of the Higher Education Commission, Thailand for supporting research grant No: KMUTNB-GOV-59-17.

\section{References}

1. Lucas, J.M.; Saccucci, M.S. Exponentially weighted moving average control schemes: properties and enhancements. Technometrics 1990, 32, 1-29.

2. Srivastava, M.S.; Wu, Y. Evaluation of optimum weights and average run lengths in EWMA control schemes. Communications in Statistics: Theory and Methods, 1997, 26, 1253-1267.

3. Roberts, S.W. Control chart tests based on geometric moving average. Technometrics 1959, 1, 239-250.

4. Crowder, S.V. A simple method for studying run length distributions of exponentially weighted moving average charts. Technometrics 1987, 29, 401-407.

5. Yashchin, E. Some aspects of the theory of statistical control schemes. IBM Journal of Research and Development 1987, 31, 199-205.

6. Ye, N.; Borror, C.; Zhang,Y. EWMA techniques for computer intrusion detection through anomalous changes in event intensity. Quality and Reliability Engineering International 2002, 18, 443-451.

7. Mastrangelo, C.M.; Montgomery, C.M. SPC with correlated observations for the chemical and process industries. Quality and Reliability Engineering International 1995, 11, 79-89.

8. VanBrackle, L.; Reynolds, M.R. EWMA and CUSUM control charts in the presence of correlation. Communications in Statistics-Simulation and Computation 1997, 26, 979-1008.

9. Harris, T.J.; Ross, W.H. Statistical process control procedures for correlated observations. The Canadian Journal of Chemical Engineering 1991, 69, 48-57.

10. Reynolds, R M.R.; Lu, C.W. Control chart for monitoring processes with autocorrelated data. Nonlinear Analysis, Theory, Methods \& Applications, 1997, 30, 4059- 4067.

11. Lu, C.W.; Reynolds, JR M.R. EWMA control charts for monitoring the mean of autocorrelated processes. Journal of Quality technology 1999, 31, 166-188. 
12. Apley, D.W.; Lee, H.C. Design of Exponentially Weighted Moving Average Control chairs for autocorrelated process with model uncertainity. Technometrics 2003, 45, 187-198.

13. Shiau, J.J.H.; Chen, Y.H. Robustness of the EWMA control chart to Non-normality for Autocorrelated process. Quality Technology \& Quantitative Management 2005, 2, 125-146.

14. Rosołowski, M.; Schmid, W. EWMA charts for monitoring the mean and the autocovariance of stationary processes. Statistical Papers 2006, 47, 595-630.

15. Mititelu, G.; Areepong,Y.; Sukparungsee, S.; Novikov, A.A. Explicit analytical solutions for the average run length of CUSUM and EWMA chart. Contribution in Mathematics and Applications II East-West Journal of Mathematics special volume 2010, 253-265.

16. suriyakat, W.; Areepong, Y.; Sukparungsee, S.; Mititelu, G. On EWMA procedure for AR(1) observations with Exponential white noise. International Journal of Pure and Applied Mathematics 2012, 77, 73-83.

17. Busaba, J.; Sukparungsee, S.; Areepong, Y.; Mititelu, G. Numerical Approximations of Average Run Length for AR(1) on Exponential CUSUM. In proceeding of the International MutiConference of Engineers and Computer Scientists, Hong Kong, 2012, 14-16 March.

18. Petcharat, K.; Areepong, Y.; Sukparungsee, S.; Mititelu, G. Exact solution of average run length of EWMA chart for MA(q) processes. Far East Journal of Mathematical Sciences 2013, 78, 291-300.

19. Phanyaem, S.; Areepong, Y.; Sukparungsee, S.; Mittitelu, G. Explicit formulas of average run length for ARMA(1,1). International Journal of Applied Mathematics and Statistics 2013, 43, 392-405.

(C) 2016 by the authors; licensee Preprints, Basel, Switzerland. This article is an open access article distributed under the terms and conditions of the Creative Commons by Attribution (CC-BY) license (http://creativecommons.org/licenses/by/4.0/). 